\title{
Identification of genes associated with renal cell carcinoma using gene expression profiling analysis
}

\author{
TING YAO $^{1 *}$, QINFU WANG $^{2 *}$, WENYONG ZHANG $^{3}$, AIHONG BIAN $^{4}$ and JINPING ZHANG ${ }^{5}$ \\ ${ }^{1}$ Physical Examination Center; Departments of ${ }^{2}$ Chronic Non-Communicable Diseases Control and Prevention, \\ ${ }^{3}$ Health Education, ${ }^{4}$ Health Inspection and ${ }^{5}$ Communicable Diseases Control and Prevention, \\ Laiwu Center for Disease Control and Prevention, Laiwu, Shandong 271100, P.R. China
}

Received March 29, 2015; Accepted April 22, 2016

DOI: $10.3892 / \mathrm{ol} .2016 .4573$

\begin{abstract}
Renal cell carcinoma (RCC) is the most common type of kidney cancer in adults and accounts for $\sim 80 \%$ of all kidney cancer cases. However, the pathogenesis of RCC has not yet been fully elucidated. To interpret the pathogenesis of RCC at the molecular level, gene expression data and bio-informatics methods were used to identify RCC associated genes. Gene expression data was downloaded from Gene Expression Omnibus (GEO) database and identified differentially coexpressed genes (DCGs) and dysfunctional pathways in RCC patients compared with controls. In addition, a regulatory network was constructed using the known regulatory data between transcription factors (TFs) and target genes in the University of California Santa Cruz (UCSC) Genome Browser (http://genome.ucsc.edu) and the regulatory impact factor of each TF was calculated. A total of 258,0427 pairs of DCGs were identified. The regulatory network contained 1,525 pairs of regulatory associations between 126 TFs and 1,259 target genes and these genes were mainly enriched in cancer pathways, ErbB and MAPK. In the regulatory network, the 10 most strongly associated TFs were FOXC1, GATA3, ESR1, FOXL1, PATZ1, MYB, STAT5A, EGR2, EGR3 and PELP1. GATA3, ERG and MYB serve important roles in RCC while FOXC1, ESR1, FOXL1, PATZ1, STAT5A and PELP1 may be potential genes associated with RCC. In conclusion, the present study constructed a regulatory network and screened out several TFs that may be used as molecular biomarkers of RCC. However, future studies are needed to confirm the findings of the present study.
\end{abstract}

Correspondence to: Dr Qinfu Wang, Department of Chronic Non-Communicable Diseases Control and Prevention, Laiwu Center for Disease Control and Prevention, 58 Luzhong East Avenue, Laicheng, Laiwu, Shandong 271100, P.R. China

E-mail: qfwang1395@163.com

*Contributed equally

Key words: renal cell carcinoma, differentially coexpressed genes, transcription factors, molecular biomarker, bioinformatics analysis

\section{Introduction}

Kidney cancer that forms in tissues of the kidney is not a single disease, instead it comprises a number of different types of cancer of which renal cell carcinoma (RCC) is the most common type in adults, responsible for $\sim 80-90 \%$ of cases $(1,2)$. The diagnosis of RCC is a challenging and difficult task, and RCC is one of the most therapy-resistant types of cancer. RCC is regarded as a highly lethal cancer and $\sim 35 \%$ of patients succumb to the disease after 5 years (3).

Understanding the pathogenesis and biological mechanism of RCC may improve the current diagnosis, treatment and prognosis of RCC. However, the pathogenesis of RCC is extremely complex and remains to be fully elucidated. Smoking, obesity and mutations in specific genes increase the risk of developing RCC (4-6). The best characterized oncogenic gene in human RCC is the tumor suppressor gene von Hippel-Lindau (VHL). VHL along with elongin B, elongin $\mathrm{C}$ and cullin 2 form a E3 ubiquitin-ligase complex, and are considered to serve an important role in $\operatorname{RCC}(7,8)$. The VHL complex targets hypoxia-inducible factor (HIF) for ubiquitin-mediated degradation (9). Mutations in VHL can result in the over accumulation of HIF, and its target genes such as VEGF (vascular endothelial growth factor), PDGF (platelet derived growth factor) and EGRF (epidermal growth factor receptor), thus resulting in carcinogenesis (10-12). Mutations of either tuberous sclerosis 1 (TSC1) or TSC2 are associated with aberrant activation of mammalian target of rapamycin (mTOR) pathway, which increases the risk of RCC $(13,14)$. Owing to the above reasons, VEGF, PDGF, EGRF and mTOR may serve as potential target molecules for the treatment of RCC. In addition, dysregulation of membrane MHC class I chain-related gene A (MICA), cyclooxygenase-1 (COX1), TGF- $\beta$-activated Kinase-1 (TAK1), and cell division cycle 25B (CDC25B) serve critical roles in RCC progression (15-18). Previous studies have predominantly focused on single genes, and paid little attention to the dysregulation of transcription factors or differentially coexpressed genes; therefore the present study aimed to address this issue to provide novel insights into RCC.

DNA microarray has previously been used to identify gene expression patterns in RCC $(19,20)$. In order to achieve a more comprehensive understanding of the molecular mecha- 
nisms underlying RCC, gene expression profiling analysis was applied to identify DCGs and regulatory network analysis was used to identify potential associations.

\section{Materials and methods}

Affymetrix microarray data. The gene expression profile dataset GSE6344 (21), including 10 RCC samples and 10 patient-matched normal control samples, was obtained from the Gene Expression Omnibus (GEO, http://www.ncbi.nlm.nih. gov/geo/) database. The probe-level data was converted into the corresponding gene symbol using the annotation information derived from platform GPL96. For genes corresponding with multiple probe sets that had multiple expression values, the mean expression values of those probe sets were obtained. As a result, 12,754 genes were obtained from this dataset.

Construction of regulatory network. The data of TF binding sites of human genome hg18 and the coordinates of genes were downloaded from UCSC (http://genome.ucsc.edu). TF binding sites within the area from $1 \mathrm{~kb}$ upstream to $0.5 \mathrm{~kb}$ downstream of the transcription initiation site of target genes were selected to construct the regulatory network.

Differentially coexpressed genes analysis. For any pair of genes $(\mathrm{X}, \mathrm{Y})$, the absolute difference (Diff) of the Pearson's correlation coefficient in the normal state (P-normal) and in the cancer state (P-tumor) was calculated. The pair of genes was regarded as DCGs only when their Diff was $>1$. Diff value $>1$ indicated that the pair of genes had a negative correlation (P-normal $\in[-1,0])$ and a positive correlation (P-tumor $\in[0,1])$ under the normal and tumor state respectively, or vice versa. The difference was calculated using the following equation 1 : Diff=abs $\left(r 1_{i j}-r 2_{i j}\right)$. In this equation, $r 1_{i j}$ and $r 2_{i j}$ indicate the Pearson correlation coefficients between gene $i$ and gene $j$ under the normal state and the state of cancer, respectively.

Measurement of RIF. Regulatory impact factors (RIF) (22), which is a robust and effective methodology to identify the regulatory impact factor of TF, was applied to identify the $\mathrm{TF}$ with the largest contribution to differential expression of genes in two biological conditions. RIF was calculated using the following equation 2 :

$$
R I F_{i}=\frac{1}{n_{d e}} \sum_{j=1}^{j=n_{d e}}\left[\left(e 1_{j} \times r 1_{i j}\right)^{2}-\left(e 2_{j} \times r 2_{i j}\right)^{2}\right]
$$

In this equation, $n_{\mathrm{de}}$ is the number of DEGs; $e 1_{\mathrm{j}}$ and $e 2 j$ indicate the expression value of the DEG in conditions 1 and 2, respectively; $r 1_{i j}$ and $r 2_{i j}$ indicate the correlation coefficient for the $i \mathrm{TF}$ and the $j \mathrm{DEG}$ in conditions 1 and 2, respectively.

Pathway enrichment analysis. For functional analysis of the large gene lists in the regulatory network, the DCGs were inputted into Database for Annotation, Visualization and Integrated Discovery (DAVID) (23) for Kyoto Encyclopedia of Genes and Genomes (KEGG) (24) pathway enrichment analysis. By calculating the hypergeometric test P-value for
Table I. Part of the differentially co-expressed genes.

\begin{tabular}{lcc}
\hline Gene1 & Gene2 & Diff \\
\hline AAGAB & A1CF & 1.031645 \\
ABCD4 & A1CF & 1.0116908 \\
ACCN2 & A1CF & 1.071472 \\
ACTR5 & A1CF & 1.039394 \\
ADAM22 & A1CF & 1.014619 \\
AHCTF1 & A1CF & 1.194273 \\
AIP & A1CF & 1.130951 \\
AK2 & A1CF & 1.069488 \\
ALKBH1 & A1CF & 1.034613 \\
AMD1 & A1CF & 1.083589 \\
AMELX & A1CF & 1.278415 \\
AMH & A1CF & 1.040918 \\
ANKRD12 & A1CF & 1.137963
\end{tabular}

Diff indicates the absolute difference of Pearson's correlation coefficient. AAGAB, $\alpha$ - and $\gamma$-adaptin binding protein; ABCD4, adenosine triphosphate binding cassette subfamily D member 4; ACCN2, acid-sensing (proton-gated) ion channel 1; ACTR5, ARP5 actinrelated protein 5 homolog (yeast);ADAM22,ADAM metallopeptidase domain 22; AHCTF1, AT-hook containing transcription factor 1; AIP, aryl hydrocarbon receptor interacting protein; AK2, adenylate kinase 2; ALKBH1, AlkB homolog 1, histone H2A dioxygenase; AMD1, adenosylmethionine decarboxylase 1; AMELX, amelogenin, X-Linked; AMH, anti-mullerian hormone; ANKRD12, ankyrin repeat domain 12; A1CF, APOBEC1 complementation factor.

probability of random association between a given list of genes and a pathway, DAVID identifies canonical pathways associated with this set of genes. FDR $<0.05$ was used as the cutoff criteria.

\section{Results}

Identification of differentially coexpressed genes in RCC. The gene expression profile dataset GSE6344 was downloaded from the GEO database and formula 1 was used to identify DCGs with Diff $>1$ between 10 RRC samples and 10 control samples. Finally, a total of 2,580,427 DCGs were screened out (Table I).

Construction of regulatory network. Based on the known regulatory data from UCSC, TFs and their corresponding target genes from DCGs were selected to construct a regulatory network. The network contained a total of 1,525 pairs of regulatory associations between $126 \mathrm{TFs}$ and 1,259 target genes. Using Cytoscape (25), the regulatory associations were integrated and visualized in Fig. 1.

KEGG pathway enrichment. The DCGs with FDR $<0.05$ were inputted into DAVID for KEGG pathway enrichment analysis. The results are presented in Table II, from which it was identified that DCGs were predominantly enriched in cancer pathways, ErbB, mitogen-activated protein kinase (MAPK) and other important pathways. 


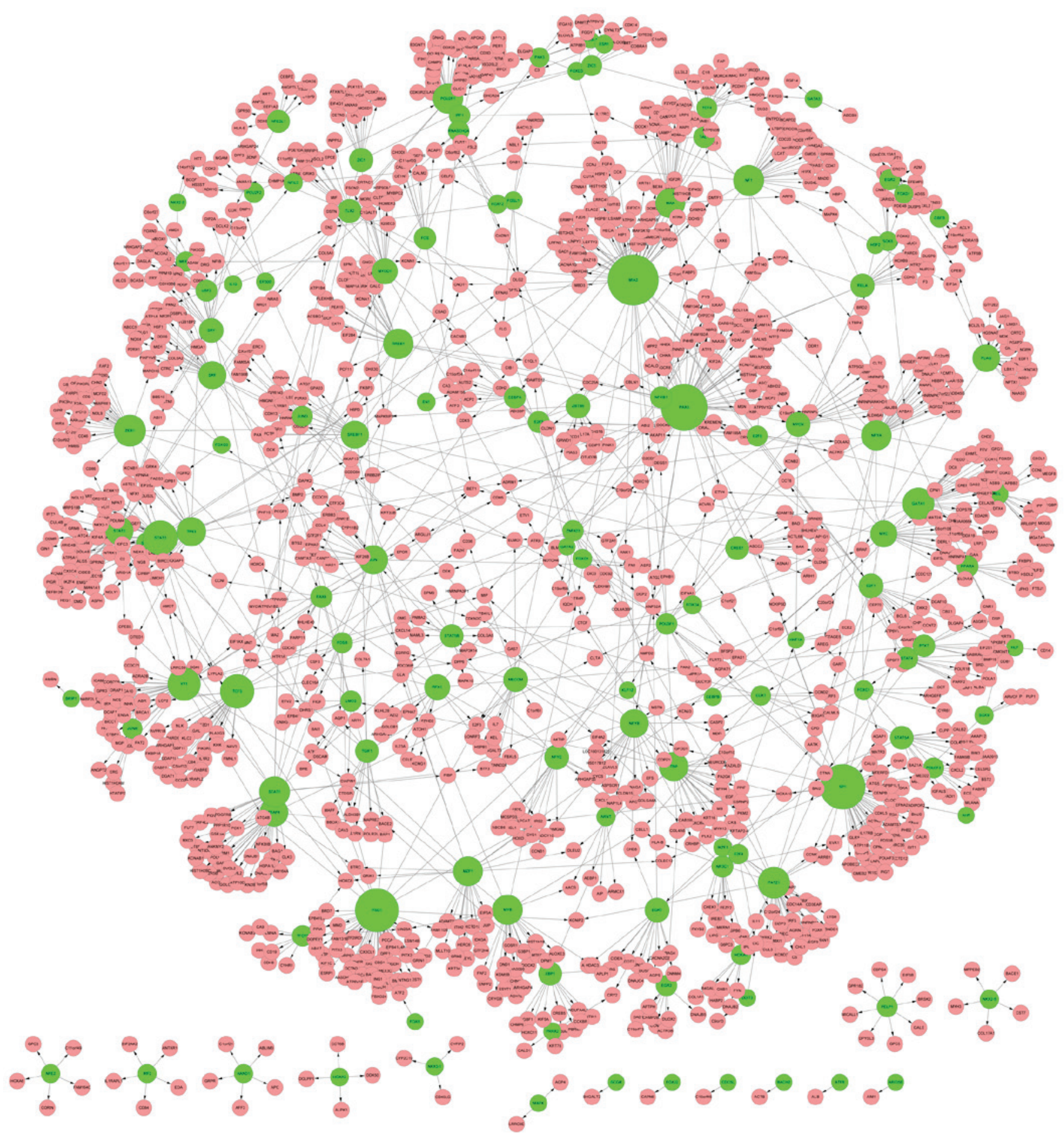

Figure 1. Regulatory network among TFs and their target genes. The green nodes indicate TF. The pink nodes indicate target genes. The lines indicate regulatory associations. TF, transcription factors.

Analysis of transcription factor impact. First, total 4,793 differentially expressed genes (DEGs) with FDR $<0.05$ were identified between normal and tumor samples by linear models for microarray data (limma) method (26). Subsequently, 469 overlapping DEGs were collected by comparing these 4,793 DEGs with the 1,259 target genes in the network. To further investigate which TFs were significant, the RIF of each TF targeting to the overlapping DEGs was targeted. The top 10 were forkhead box C1 (FOXC1), GATA-binding protein 3 (GATA3), estrogen receptor 1 (ESR1), FOXL1, POZ
(BTB) and AT hook containing zinc finger 1 (PATZ1), v-myb avian myeloblastosis viral oncogene homolog (MYB), signal transducer and activator of transcription 5A (STAT5A), early growth response 2 (EGR2), EGR3 and proline, glutamate and leucine rich protein 1 (PELP1) (Table III). Of these TFs, GATA3, MYB, EGR2, and EGR3 have previously been identified to be associated with RCC and the regulatory associations of them with their targets are presented in Fig. 2. Occurrence of RCC is likely caused by the abnormal changes of these regulatory associations. 
Table II. The enriched KEGG pathways.

\begin{tabular}{llr}
\hline Category & \multicolumn{1}{c}{ Term } & FDR (\%) \\
\hline KEGG PATHWAY & has05200:Pathways in cancer & 0.012504 \\
KEGG PATHWAY & has05215:Prostate cancer & 0.028298 \\
KEGG PATHWAY & has04710:Circadian rhythm & 0.147888 \\
KEGG PATHWAY & has04012:ErbB singling pathway & 0.185805 \\
KEGG PATHWAY & has05220:Chronic myeloid leukemia & 0.192832 \\
KEGG PATHWAY & has05221:Acute myeloid leukemia & 0.218097 \\
KEGG PATHWAY & has05222:Small cell lung cancer & 0.323675 \\
KEGG PATHWAY & has05212:Pancreatic cancer & 1.018255 \\
KEGG PATHWAY & has04010:MAPK singling pathway & 1.268065 \\
KEGG PATHWAY & has05214:Glioma & 1.84753 \\
KEGG PATHWAY & has05213:Endometrial singling pathway & 2.33947 \\
KEGG PATHWAY & has04062:Chemokine singling pathway & 3.258893 \\
KEGG PATHWAY & has05120:Epithelial cellsignaling in Helicobacter pylori infection & 4.078908
\end{tabular}

KEGG, Kyoto Encyclopedia of Genes and Genomes.

Table III. The top 10 ranked TFs.

\begin{tabular}{llc}
\hline TF & RIF score & RIF rank \\
\hline FOXC1 & 7.804438725 & 1 \\
GATA3 & 6.908779522 & 2 \\
ESR1 & 6.32301186 & 3 \\
FOXL1 & 4.242514268 & 4 \\
PATZ1 & 3.800727043 & 5 \\
MYB & 3.507929833 & 6 \\
STAT5A & 3.467483166 & 7 \\
EGR2 & 3.361578969 & 8 \\
EGR3 & 3.337751915 & 9 \\
PELP1 & 2.818195935 & 10 \\
\hline
\end{tabular}

TF represents the transcription factor in the regulatory network. RIF represents the regulatory impact factor of TF. Rank represents the impact rank of TF. TF, transcription factor; FOXC1, forkhead box $\mathrm{C} 1$; GATA3, GATA-binding protein 3; ESR1, estrogen receptor 1, FOXL1, forkhead box L1; PATZ1, POZ (BTB) and AT hook containing zinc finger 1; MYB, v-myb avian myeloblastosis viral oncogene homolog, STAT5A, signal transducer and activator of transcription 5A, EGR2/3, early growth response 2 or 3; PELP1, proline, glutamate and leucine rich protein 1 .

\section{Discussion}

Molecular biomarkers are useful to improve diagnosis, clinical predictive capability and novel therapeutic efficacy. Because the emergence of microarray technology makes it possible to investigate the expression levels of thousands of genes simultaneously (27), it has been widely used in discovery of disease biomarkers (28-30). However, the majority of previous studies are based on a single gene differential expression analysis. In the current study, differentially coexpressed genes analysis was used to find the dysregulated gene pairs in RCC. The differ- entially coexpressed genes analysis provides novel analytical perspective to studies including identification of differentially coexpressed genes and marker genes of disease, construction of differential coexpression network and analysis of module $(31,32)$. Besides, a regulatory network was constructed and the RIF score of each TF targeting to the DEGs with FDR $<0.05$ was calculated. Finally, the top 10 ranked TFs were identified which were FOXC1, GATA3, ESR1, FOXL1, PATZ1, MYB, STAT5A, EGR2, EGR3 and PELP1. They may be potential molecular biomarkers of RCC.

FOXC1 is one of the forkhead transcription factor family, which is characterized by a unique DNA-binding domain. Although the specific function of this gene has not yet been determined, it is known that the gene is associated with the occurrence of a number of types of cancer, such as breast cancer, lung cancer, prostate cancer, bile duct cancer (33-36). FOXC1 appears to be a cancer-associated gene. FOXL1 and FOXC1 belong to the same family and have similar functions. So these genes may serve important roles in the pathogenesis of RCC.

The transcription factor encoding by GATA3 belongs to the GATA family, and it contains two GATA-type zinc fingers which serve an important role in regulating $\mathrm{T}$ cell development and the biology of endothelial cell. Cooper et al (37) demonstrated that GATA3 was methylated in clear cell RCC patients and its mRNA expression level was downregulated in all stages of clear cell RCC (37), which indicated the critical role of GATA3 in RCC.

As an estrogen receptor, ESR1, which contains a number of important structural domains such as the DNA-binding domain, transcriptional activation domain and hormone binding domain, is a TF activated by its corresponding ligand. It is known that ESR1 serves an important role in breast cancer (38). Although it has not yet been reported that this gene is associated with RCC at present, it is likely to be a potential factor because its RIF ranks third.

Among PATZ1, MYB, STAT5A, EGR2/EGR3 and PELP1, it is known that MYB and EGR2/EGR3 (39) are RCC associ- 

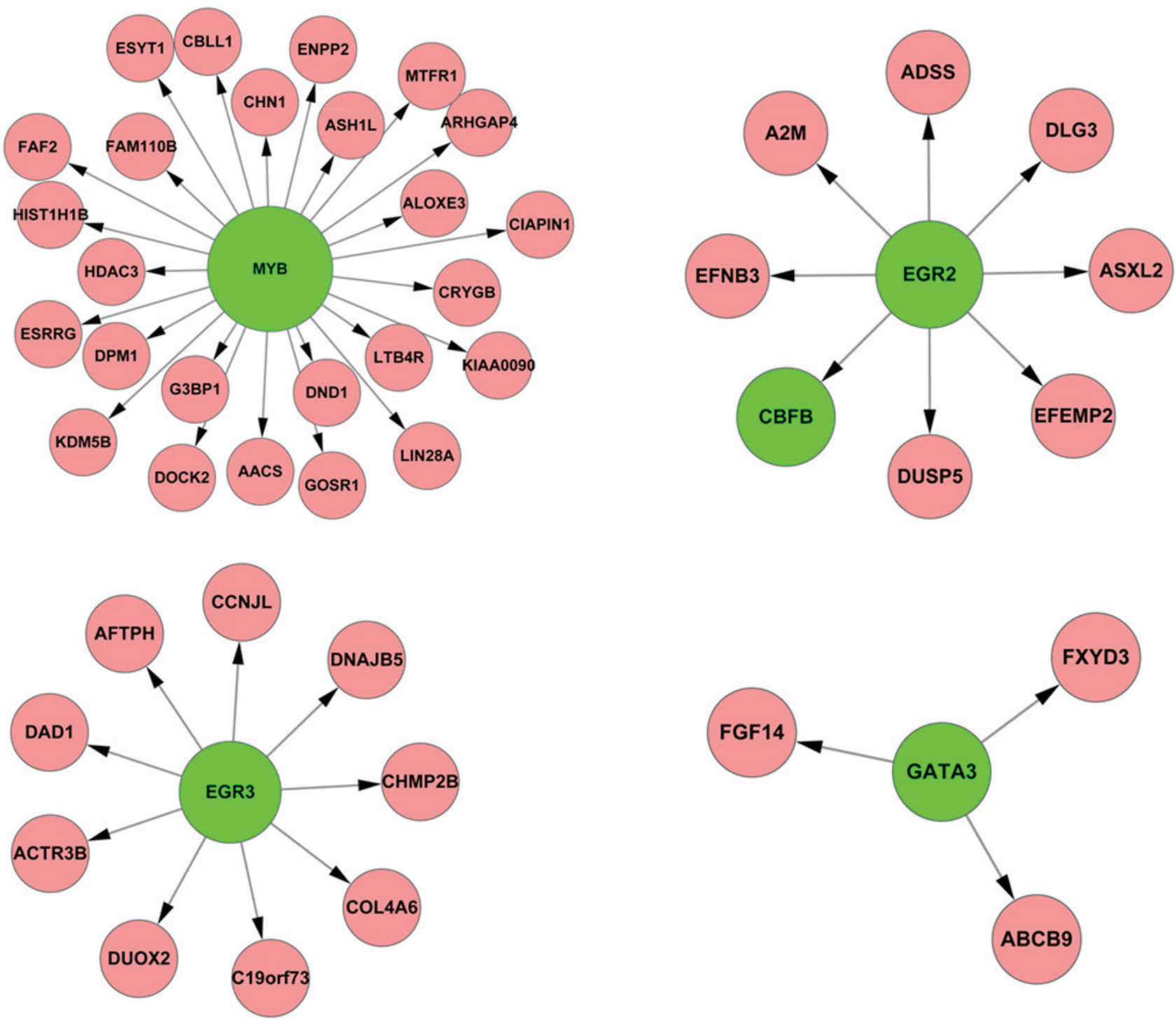

Figure 2. The regulatory associations between the $4 \mathrm{TFs}$ associated with RRC and their target genes. The green nodes indicate TFs and the red nodes indicate their target genes. TF, transcription factors; RCC, renal cell carcinoma.

ated genes, and the PATZ1, STAT5A and PELP1 are hormone associated genes. The latest article reported that hormones may inhibit the occurrence of renal carcinoma (40), so that PATZ1, STAT5A and PELP1 may serve as potential renal cancerassociated genes.

Table II identifies genes of the regulatory network which were enriched in various cancer pathways, just as the above discussion that the top 10 TFs were involved in a number of types of cancers. In addition, ErbB and MAPK pathways were overrepresented. The MAPK signaling pathways serve vital roles in cell proliferation and differentiation. Recently, Huang et al (41) reported that suppression of one or more MAPK signaling pathways by inhibitor of MAPK kinases (MKKs) reduced RCC cell proliferation in vitro and inhibited RCC growth in vivo. The ErbB protein family is a family containing 4 structurally associated receptor tyrosine kinases, ErbB1 (also termed EGFR), ErbB2, ErbB3 and ErbB4. ErbB signaling pathway has been implicated in the development of a wide variety of types of tumor, including RCC $(42,43)$. Further analysis of these pathways will contribute to an improved understanding of the roles of the differentially expressed genes and the underlying molecular mechanism of RCC.

In conclusion, the present study used microarray gene expression profiling and regulatory network analysis to explore the molecular mechanism of RCC. The top 10 ranked TFs were identified, which were FOXC1, GATA3, ESR1, FOXL1, PATZ1, MYB, STAT5A, EGR2, EGR3 and PELP1. GATA3, ERG and MYB are considered RCC associated genes while FOXC1, ESR1, FOXL1, PATZ1, STAT5A and PELP1 may also be potential genes associated with RCC. The present study indicates that the above TFs may be used as biomarkers of RCC for accurate diagnosis, prognosis or as predictive markers for treatment efficiency. However, further experiments are needed to confirm these result.

\section{References}

1. Yi Z, Fu Y, Zhao S, Zhang X and Ma C: Differential expression of miRNA patterns in renal cell carcinoma and nontumorous tissues. J Cancer Res Clin Oncol 136: 855-862, 2010. 
2. Ljungberg B, Campbell SC, Cho HY, Jacqmin D, Lee JE, Weikert $S$ and Kiemeney LA: The epidemiology of renal cell carcinoma. Eur Urol 60: 615-621, 2011.

3. Lipworth L, Tarone RE and McLaughlin JK: The epidemiology of renal cell carcinoma. J Urol 176: 2353-2358, 2006.

4. Beebe-Dimmer JL, Colt JS, Ruterbusch JJ, Keele GR, Purdue MP, Wacholder S, Graubard BI, Davis F, Chow WH and Schwartz KL: Body mass index and renal cell cancer: The influence of race and sex. Epidemiology 23: 821-828, 2012.

5. Linehan WM, Srinivasan R and Schmidt LS: The genetic basis of kidney cancer: A metabolic disease. Nat Rev Urol 7: 277-285, 2010.

6. Kroeger N, Klatte T, Birkhäuser FD, Rampersaud EN Seligson DB, Zomorodian N, Kabbinavar FF, Belldegrun AS and Pantuck AJ: Smoking negatively impacts renal cell carcinoma overall and cancer-specific survival. Cancer 118: 1795-1802, 2012.

7. Gnarra J, Tory K, Weng Y, Schmidt L, Wei MH, Li H, Latif F, Liu S, Chen F and Duh FM: Mutations of the VHL tumour suppressor gene in renal carcinoma. Nat Genet 7: 85-90, 1994.

8. Störkel S, Eble JN, Adlakha K, Amin M, Blute ML, Bostwick DG Darson M, Delahunt B and Iczkowski K: Classification of renal cell carcinoma: Workgroup No. 1. union internationale contre le cancer (UICC) and the american joint committee on cancer (AJCC). Cancer 80: 987-989, 1997.

9. Krek W: VHL takes HIF's breath away. Nat Cell Biol 2: E121-E123, 2000.

10. Kim WY and Kaelin WG: Role of VHL gene mutation in human cancer. J Clin Oncol 22: 4991-5004, 2004.

11. Wiesener MS, Münchenhagen PM, Berger I, Morgan NV, Roigas J, Schwiertz A, Jürgensen JS, Gruber G, Maxwell PH, Löning SA, et al: Constitutive activation of hypoxia-inducible genes related to overexpression of hypoxia-inducible factor-1alpha in clear cell renal carcinomas. Cancer Res 61: 5215-5222, 2001.

12. Kaelin WG Jr: Molecular basis of the VHL hereditary cancer syndrome. Nat Rev Cancer 2: 673-682, 2002.

13. Kenerson HL, Aicher LD, True LD and Yeung RS: Activated mammalian target of rapamycin pathway in the pathogenesis of tuberous sclerosis complex renal tumors. Cancer Res 62: $5645-5650,2002$.

14. Sampson JR: Therapeutic targeting of mTOR in tuberous sclerosis. Biochem Soc Trans 37: 259-264, 2009.

15. Jia HY, Liu JL, Zhou CJ, Kong F, Yuan MZ, Sun WD, Wang J, Liu L, Zhao JJ and Luan Y: High expression of MICA in human kidney cancer tissue and renal cell carcinoma lines. Asian Pac J Cancer Prev 15: 1715-1717, 2014.

16. Yu ZH, Zhang Q, Wang YD, Chen J, Jiang ZM, Shi M, Guo X, Qin J, Cui GH, Cai ZM, et al: Overexpression of cyclooxygenase-1 correlates with poor prognosis in renal cell carcinoma. Asian Pac J Cancer Prev 14: 3729-3734, 2013.

17. Wei C, Lai YQ, Li XX and Ye JX: TGF- $\beta$-activated kinase-1: A potential prognostic marker for clear cell renal cell carcinoma. Asian Pac J Cancer Prev 14: 315-320, 2013.

18. Yu XY, Zhang Z, Zhang GJ, Guo KF and Kong CZ: Knockdown of Cdc25B in renal cell carcinoma is associated with decreased malignant features. Asian Pac J Cancer Prev 13: 931-935, 2012.

19. Seliger B, Dressler SP, Wang E, Kellner R, Recktenwald CV, Lottspeich F, Marincola FM, Baumgärtner M, Atkins D and Lichtenfels R: Combined analysis of transcriptome and proteome data as a tool for the identification of candidate biomarkers in renal cell carcinoma. Proteomics 9: 1567-1581, 2009.

20. Seliger B, Dressler SP, Lichtenfels R and Kellner R: Candidate biomarkers in renal cell carcinoma. Proteomics 7: 4601-4612, 2007.

21. Tun HW, Marlow LA, von Roemeling CA, Cooper SJ, Kreinest $P$, Wu K, Luxon BA, Sinha M, Anastasiadis PZ and Copland JA: Pathway signature and cellular differentiation in clear cell renal cell carcinoma. PLos One 5: e10696, 2010.

22. Reverter A, Hudson NJ, Nagaraj SH, Pérez-Enciso M and Dalrymple BP: Regulatory impact factors: Unraveling the transcriptional regulation of complex traits from expression data. Bioinformatics 26: 896-904, 2010.

23. Dennis G Jr, Sherman BT, Hosack DA, Yang J, Gao W, Lane HC and Lempicki RA: DAVID: Database for annotation, visualization and integrated discovery. Genome Biol 4: P3, 2003.
24. Kanehisa M: The KEGG database. Novartis Found Symp 247 91-101; discussion 101-103, 2002.

25. Shannon P, Markiel A, Ozier O, Baliga NS, Wang JT, Ramage D, Amin N, Schwikowski B and Ideker T: Cytoscape: A software environment for integrated models of biomolecular interaction networks. Genome Res 13: 2498-2504, 2003.

26. Smyth GK: Linear models and empirical bayes methods for assessing differential expression in microarray experiments. Stat Appl Genet Mol Biol 3: Article3, 2004.

27. King HC and Sinha AA: Gene expression profile analysis by DNA microarrays: Promise and pitfalls. JAMA 286: 2280-2288, 2001.

28. Guttula SV, Allam A and Gumpeny RS: Analyzing microarray data of alzheimer's using cluster analysis to identify the biomarker genes. Int J Alzheimers Dis 2012: 649456, 2012.

29. Wu MY, Dai DQ, Shi Y, Yan H and Zhang XF: Biomarker identification and cancer classification based on microarray data using Laplace naive bayes model with mean shrinkage. IEEE/ACM Trans Comput Biol Bioinform 9: 1649-1662, 2012.

30. Arias CR, Yeh HY and Soo VW: Biomarker identification for prostate cancer and lymph node metastasis from microarray data and protein interaction network using gene prioritization method. Scientific World Journal 2012: 842727, 2012

31. Lai Y: Differential expression analysis of digital gene expression data: RNA-tag filtering, comparison of t-type tests and their genome-wide co-expression based adjustments. Int J Bioinform Res Appl 6: 353-365, 2010.

32. Choi JK, Yu U, Yoo OJ and Kim S: Differential coexpression analysis using microarray data and its application to human cancer. Bioinformatics 21: 4348-4355, 2005.

33. Wei LX, Zhou RS, Xu HF, Wang JY and Yuan MH: High expression of FOXC1 is associated with poor clinical outcome in non-small cell lung cancer patients. Tumour Biol 34: 941-946, 2013.

34. Sizemore ST and Keri RA: The forkhead box transcription factor FOXC1 promotes breast cancer invasion by inducing matrix metalloprotease 7 (MMP7) expression. J Biol Chem 287: 24631-24640, 2012.

35. Chung TK, Lau TS, Cheung TH, Yim SF, Lo KW, Siu NS, Chan LK, Yu MY, Kwong J, Doran G, et al: Dysregulation of microRNA-204 mediates migration and invasion of endometrial cancer by regulating FOXC1. Int J Cancer 130: 1036-1045, 2012.

36. Muggerud AA, Rønneberg JA, Wärnberg F, Botling J, Busato F, Jovanovic J, Solvang H, Bukholm I, Børresen-Dale AL, Kristensen VN, et al: Frequent aberrant DNA methylation of ABCB1, FOXC1, PPP2R2B and PTEN in ductal carcinoma in situ and early invasive breast cancer. Breast Cancer Res 12: R3, 2010.

37. Cooper SJ, Zou H, Legrand SN, Marlow LA, von Roemeling CA, Radisky DC, Wu KJ, Hempel N, Margulis V, Tun HW, et al: Loss of type III transforming growth factor-beta receptor expression is due to methylation silencing of the transcription factor GATA3 in renal cell carcinoma. Oncogene 29: 2905-2915, 2010.

38. Sommer S and Fuqua SA: Estrogen receptor and breast cancer. Semin Cancer Biol 11: 339-352, 2001.

39. Amatschek S, Koenig U, Auer H, Steinlein P, Pacher M, Gruenfelder A, Dekan G, Vogl S, Kubista E, Heider KH, et al: Tissue-wide expression profiling using cDNA subtraction and microarrays to identify tumor-specific genes. Cancer Res 64: 844-856, 2004.

40. Yu CP, Ho JY, Huang YT, Cha TL, Sun GH, Yu DS, Chang FW, Chen SP and Hsu RJ: Estrogen inhibits renal cell carcinoma cell progression through estrogen receptor- $\beta$ activation. PLos One 8: e56667, 2013

41. Huang D, Ding Y, Luo WM, Bender S, Qian CN, Kort E, Zhang ZF, VandenBeldt K, Duesbery NS, Resau JH and Teh BT: Inhibition of MAPK kinase signaling pathways suppressed renal cell carcinoma growth and angiogenesis in vivo. Cancer Res 68 81-88, 2008.

42. Hynes NE and MacDonald G: ErbB receptors and signaling pathways in cancer. Curr Opin Cell Biol 21: 177-184, 2009.

43. Weber KL, Doucet M, Price JE, Baker C, Kim SJ and Fidler IJ: Blockade of epidermal growth factor receptor signaling leads to inhibition of renal cell carcinoma growth in the bone of nude mice. Cancer Res 63: 2940-2947, 2003. 